\title{
Funções Executivas, Atenção e o Uso do Metilfenidato: Estudo de Revisão Sistemática
}

\author{
Lara Fechine Piquet da Cruz (๑),Carla Alexandra da Silva Moita Minervino (®), \\ \& Estephane Enadir Lucena Duarte Pereira 1 \\ Universidade Federal da Paraíba, João Pessoa, PB, Brasil
}

\begin{abstract}
RESUMO - A influência do metilfenidato no desempenho das funções executivas e atenção tem sido bastante estudada no transtorno do déficit de atenção/hiperatividade. Este artigo consiste em um estudo de revisão sistemática de pesquisas publicadas entre 2010 e 2016 e que envolvem a verificação do perfil de desempenho em funções executivas e atenção de crianças e/ou adolescentes com TDAH que usam ou não metilfenidato. Foram utilizados os descritores executive functions AND methylphenidate, adhd, attention. A análise dos artigos revelou que o metilfenidato é considerado a substância de primeira escolha para o TDAH e que sua eficácia é mais comprovada no controle inibitório e na flexibilidade cognitiva. São necessários mais estudos no que se refere à memória de trabalho e atenção.
\end{abstract}

PALAVRAS-CHAVES: funções executivas, metilfenidato, revisão sistemática

\section{Executive Functions, Attention, and the Use of Methylphenidate: A Systematic Review Study}

\begin{abstract}
The influence of methylphenidate in performance of executive functions and attention has been much studied on attention deficit/hyperactivity disorder. This article consists of a systematic review study of public researches between 2010 and 2016 that involves a verification of the performance profile on executive functions and attention of children and/or adolescents with ADHD, using methylphenidate or not. As descriptors, the following expressions were used: executive functions AND methylphenidate, adhd, attention. The analysis of the articles revealed that methylphenidate is considered a first-choice substance for ADHD and that its efficacy is better demonstrated on inhibitory control and cognitive flexibility. There is a need for more studies regarding working memory and attention.
\end{abstract}

KEYWORDS: executive functions, methylphenidate, systematic review

O transtorno do déficit de atenção/hiperatividade (TDAH), de acordo com o Manual Diagnóstico e Estatístico de Transtornos Mentais (DSM-5) de 2013, classifica-se entre os transtornos do neurodesenvolvimento caracterizados por dificuldades no desenvolvimento que se manifestam precocemente e influenciam o funcionamento pessoal, social ou acadêmico do indivíduo. Um padrão persistente de desatenção e/ou hiperatividade-impulsividade interfere no funcionamento ou no desenvolvimento e, frequentemente, vincula-se a comorbidades como transtorno de aprendizagem, transtorno de conduta, depressão, transtorno de ansiedade ou transtorno desafiador e opositor (American Psychiatric Association, 2013; Bolfer, 2014).
O DSM-5 estabelece três apresentações do TDAH: TDAH apresentação combinada (TDAH-C), em que o sujeito tem ambos os conjuntos de critérios comportamentais estabelecidos tanto para a desatenção quanto para a hiperatividade-impulsividade (durante, pelo menos, seis meses); TDAH apresentação predominantemente desatenta (TDAH-D), em que o sujeito se insere em, pelo menos, seis dos critérios estabelecidos para a desatenção, e $T D A H$ apresentação predominantemente hiperativa-impulsiva (TDAH-H), em que o sujeito se encaixa em, pelo menos, seis dos critérios para essas características (Rohde \& Halpern, 2004; Whitbourne \& Halgin, 2015).

\footnotetext{
*E-mail: larafpiquet@hotmail.com

- Submetido: 01/06/2017; Aceito: 06/08/2018.
} 
Em suma, o TDAH é um dos distúrbios neuropsicológicos mais comuns, que inicia na primeira infância, afeta de 3 a $7 \%$ das crianças em idade escolar e é um dos quadros de mais interesse em avaliação neuropsicológica infantil. $\mathrm{O}$ transtorno tem como principais características sintomas de desatenção, hiperatividade, impulsividade e prejuízos cognitivos, principalmente nas funções executivas (FE's), que afetam o desempenho escolar e as relações interpessoais do sujeito. De acordo com sua fisiopatologia, o TDAH parece envolver alterações nas vias dopaminérgicas e noradrenérgicas, relacionadas ao controle de atenção e de impulsividade (Gonçalves et al., 2013; Huang et al., 2012; Pasini et al., 2013; Yang et al., 2012).

\section{TDAH e o Déficit nas Funções Executivas}

Devido à alteração no funcionamento executivo, o transtorno, muitas vezes, é caracterizado como transtorno da disfunção executiva. Funções executivas são habilidades cognitivas por meio das quais o sujeito pode se engajar em comportamentos direcionados a metas; portanto, permitem que o sujeito monitore, planeje e realize comportamentos intencionais e com objetivos definidos (Diamond, 2013; Dias, 2009; Menezes et al., 2012).

As FE's iniciam o seu desenvolvimento na infância e evoluem até a fase adulta de uma forma lenta que acompanha a maturação do córtex pré-frontal (CPF), à medida que essas funções vão se aperfeiçoando. Fala-se em um desenvolvimento no formato de $\mathrm{U}$ invertido, visto que o pico da maturação ocorre no fim da adolescência, estabiliza-se na vida adulta, e sua eficácia diminui ao longo do envelhecimento. As FE's estão localizadas no CPF e, como os pacientes com TDAH têm alteração nesta área que está ligada a um bom funcionamento executivo, explica-se o fato de as pessoas com o transtorno terem déficit nas FE's (Fuentes et al., 2014; Menezes et al., 2012; Pires, 2014).

De acordo com o modelo de Diamond (2013), as FE's têm três habilidades principais: a de controle inibitório, a de memória de trabalho e a de flexibilidade cognitiva. Todas as outras habilidades (planejamento, raciocínio, tomada de decisão, resolução de problemas) emergem dessas três habilidades principais (Diamond, 2013; Dias et al., 2010; Fuentes et al., 2014; Menezes et al., 2012; Schoemaker et al., 2013). O modelo de Diamond (2013) parte da teoria de Miyake et al. (2000) e está ligado ao chamado Modelo Fatorial das funções executivas, que defende a ideia de que os fatores são separados, e não, que só há um fator para essas habilidades cognitivas.

A memória de trabalho é a capacidade de adquirir novas informações, mantê-las em mente e trabalhar com elas. $\mathrm{O}$ controle inibitório se refere à capacidade de inibir comportamentos, pensamentos ou emoções inadequados para determinadas situações, o que, consequentemente, substitui a pré-disposição a fazer o que não é adequado no determinado momento. A flexibilidade cognitiva está relacionada a ser capaz de mudar de perspectiva rapidamente e se adaptar à situação que está presente e que não pode ser modificada (Diamond, 2013; Fuentes et al., 2014; Menezes et al., 2012; Whitbourne \& Halgin, 2015).

\section{TDAH e o Déficit na Atenção}

Além do déficit nas FE's, pacientes com TDAH têm déficit em outra habilidade cognitiva, a atenção, que está relacionada à seleção, à filtragem e à organização de estímulos que se originam do meio externo ou interno e é subjacente a todo o processo cognitivo. Ela é considerada um fator de proteção do organismo, porque protege o indivíduo da grande quantidade de estímulos que recebe constantemente do meio (Bolfer, 2014; Capovilla \& Dias, 2008; Fuentes et al., 2014; Menezes et al., 2012; Posner, 2012). A atenção classifica-se em quatro tipos: seletiva, sustentada, alternada e dividida, de acordo com o processamento em que está envolvida. Esses mecanismos atencionais estão associados, apesar de terem funções distintas (Bolfer, 2009; Bolfer, 2014; Capovilla \& Dias, 2008; Nardi et al., 2015).

A atenção seletiva consiste em selecionar estímulos importantes e inibir os que são distratores; a atenção sustentada envolve a manutenção do foco atencional em uma mesma atividade por um período de tempo extenso; a atenção alternada consiste em mudar o foco atencional de um estímulo para outro, não simultaneamente, mas alternadamente; e a atenção dividida requer que se divida, ao mesmo tempo, a atenção entre estímulos em tarefas independentes, uma executada mais automaticamente do que a outra (Bolfer, 2009; Bolfer, 2014; Copovilla \& Dias, 2008; Menezes et al., 2012).

\section{Tratamento do TDAH: Metilfenidato}

O tratamento de pacientes com TDAH é feito, principalmente, com o uso de medicamentos psicoestimulantes. Os mais utilizados são os remédios à base do metilfenidato (MPH). O MPH tem como perfil farmacológico impedir que a dopamina e a noradrenalina sejam recapturadas pelos neurônios pré-sinápticos. Assim, aumenta a concentração desses neurotransmissores na fenda sináptica e alivia os sintomas do transtorno (Bezerra, 2014; Bolfer, 2014; Ferreira, 2006; Fuentes et al., 2014; Whitbourne \&Halgin, 2015).

$\mathrm{O} \mathrm{MPH}$ é administrado via oral e pode ser liberado no organismo de maneiras diferentes. Para que essa liberação seja imediata, o MPH precisa ser tomado três vezes por dia, para garantir que a eficácia terapêutica seja mantida. Porém o comprimido também pode ser liberado de forma prolongada no organismo, e o efeito pode durar até 12 horas. Para isso, pode ser tomado uma vez por dia. No MPH de liberação prolongada, usa-se a tecnologia denominada de sistema de liberação osmótica oral (OROS), e este é chamado 
de OROS-metilfenidato (Agência Nacional de Vigilância Sanitária [ANVISA], 2014).

$\mathrm{Na}$ Europa e na América do Norte, a atomoxetina (ATX) também é um fármaco usado para o tratamento do TDAH. AATX é um inibidor da receptação pré-sináptica da noradrenalina e, comparado com o efeito nesse transportador, tem pouca afinidade para transportadores de serotonina ou dopamina. Têm sido discutidos os efeitos positivos de psicoestimulantes nas funções executivas no TDAH, mas ainda é incerto se a ATX causa efeito no funcionamento neuropsicológico em crianças com o transtorno (Taracena \& Rada, 2007).

O metilfenidato (MPH) exerce efeitos de tratamento tanto para a dimensão cognitiva quanto comportamental em pacientes com TDAH e tem havido um crescente aumento do consumo desse medicamento, que pode ser devido ao aumento das prescrições do MPH para tratar o transtorno ou do uso sem prescrição por parte de pessoas que buscam melhora cognitiva (ANVISA, 2014).

Há debates sobre o aumento das prescrições indiscriminadas do MPH. A ANVISA (2014), por exemplo, pontua o maior número de diagnósticos de TDAH ou os diagnósticos imprecisos do transtorno como motivo desse aumento de prescrições e do consumo sem prescrição. Itaborahy e Ortega (2013) analisaram publicações brasileiras sobre o uso do MPH e encontraram 35 reportagens que retratam um excesso de prescrições do medicamento que ocorreriam porque todos os casos de TDAH em crianças estariam sendo medicados, até mesmo sem necessidade, ou porque crianças sem esse transtorno estariam recebendo a medicação.

Ademais, têm surgido controvérsias acerca do uso, dos benefícios e da eficácia do MPH para crianças e/ou adolescentes com TDAH. Uma das discussões diz respeito a um dos efeitos adversos do MPH, que é o risco de abuso e de dependência. Segundo a ANVISA (2014), o MPH tem um alto potencial de risco e dependência. Moysés e Collares (2010, citados por Toassa, 2012), pontuam que o MPH tem o mesmo mecanismo de ação da cocaína, por isso, um dos riscos da utilização dessa medicação em longo prazo seria a adição de cocaína. Em contrapartida, autores como Ortega et al. (2010) e Itaborahy e Ortega (2013) citam o termo antidependência, ao afirmar que esse seria um benefício do uso do $\mathrm{MPH}$, visto que o medicamento não causaria dependência e evitaria o abuso de substâncias no futuro, que é um risco causado pelo próprio TDAH.

Outros efeitos adversos causados por esse medicamento, que são os mais relatados na maioria dos artigos pesquisados no estudo de Itaborahy e Ortega (2013) e de acordo com a ANVISA (2014), são diminuição do apetite, dores de cabeça, insônia, perda de peso, dores abdominais e redução do crescimento.

Em relação aos benefícios e à eficácia do metilfenidato no tratamento do TDAH, estudos, que serão citados a seguir, apontam conclusões diferentes. Itaborahy e Ortega (2013) pontuam benefícios como a melhoria na qualidade do sono, baixa toxidade, segurança clínica e superioridade, quando comparado com o placebo ou com a psicoterapia. Ortega et al. (2010) também apresentam considerações acerca do benefício da boa tolerância desse medicamento. Contrariamente à ideia de superioridade do $\mathrm{MPH}$, quando comparado com a psicoterapia, autores como Bezerra (2014) e Bolfer (2014) relatam que o tratamento mais eficaz do TDAH é o que associa o uso do MPH com psicoterapia.

Acerca da eficácia do metilfenidato, Itaborahy e Ortega (2013) e Ortega et al. (2010) mencionam a redução dos sintomas do TDAH que, consequentemente, melhora o desempenho acadêmico. Outros autores, como Bezerra (2014), Bolfer (2014), Brant e Carvalho (2012), Ferreira (2006), Maneeton et al. (2015) e Murray et al. (2011), asseveram que, além de diminuir os sintomas do transtorno, melhora as habilidades atencionais e as habilidades de funções executivas. Porém, de acordo com Andrade e Scheuer (2004), há autores estrangeiros cujos estudos revelaram que, em média, um quarto das crianças com TDAH não apresentam melhora ao usarem MPH ou que 20,4\% delas não são beneficiadas e podem até piorar.

No artigo de Silva et al. (2012) é feita uma revisão bibliográfica de estudos sobre o consumo do MPH, especificamente da ritalina, e os autores concluíram que a eficácia desse medicamento é questionável. E como a ritalina é utilizada, primeiramente, para eliminar as queixas ligadas ao TDAH e retém a hiperatividade da criança por um tempo, isso tranquiliza seus pais, que passam a considerar o medicamento como eficaz. Os autores supracitados também criticam a forma como tem sido feito o diagnóstico do transtorno, porque só é baseado nos sintomas, o que tem sido o foco do tratamento, segundo a visão da medicina atual. Para os autores, o diagnóstico baseado só em sintomas e o uso "indiscriminado e indevido" do medicamento contribuem para o aumento do consumo do MPH.

Considerando-se a relevância de conhecer mais sobre o TDAH e seu tratamento, com o objetivo de reduzir os sintomas e os riscos causados pelo transtorno, e considerando-se as controvérsias dos estudos que tratam do uso do metilfenidato, entende-se a importância de realizar esta revisão sistemática de pesquisas que envolvem a análise neuropsicológica do perfil de desempenho em funções executivas e atenção de crianças e/ou adolescentes com TDAH em uso do metilfenidato.

Foi encontrada uma revisão sistemática realizada por Storebo et al. (2015) que também trata dessa temática, porém a pergunta geradora é diferente, pois este estudo analisa o perfil de funcionamento executivo e atencional de crianças e adolescentes com TDAH que usam o metilfenidato, enquanto a revisão dos autores supracitados analisa os malefícios e os benefícios desse medicamento no comportamento de crianças e adolescentes com TDAH, e essa discussão não entra neste estudo. Contudo, esta revisão sistemática versa sobre a análise dos aspectos metodológicos das pesquisas, com ênfase na discussão crítica dos resultados e, principalmente, na busca por evidências da magnitude da relação entre o metilfenidato e o desempenho em funções executivas e atenção. 


\section{METODOLOGIA}

A revisão sistemática foi iniciada a partir da questão geradora: A utilização do metilfenidato relaciona-se positivamente com o desempenho em funções executivas e atenção? A pesquisa e a análise do material foram conduzidas em 2016 em quatro fases, depois de verificados os critérios de elegibilidade.

Na primeira fase, foram selecionadas as seguintes bases de dados eletrônicas: Medline, Web of Science, Scopus, Elsevier, PsycINFO, Science Direct, Lilacs, BVS-PSI e EBSCO e utilizados como descritores os termos executive functions AND methylphenidate; adhd; attention. Na busca, considerou-se a opção "todos os campos", mas não se fez qualquer tipo de seleção por título ou autor.

$\mathrm{Na}$ segunda fase, foram estabelecidos os critérios de inclusão, a saber: (a) artigos de pesquisa empírica escritos em língua inglesa; (b) amostras de crianças e/ou adolescentes com TDAH; (c) amostras de crianças e/ou adolescentes em uso ou não de metilfenidato; (d) artigo que responde à pergunta geradora do objetivo central desta investigação; (e) artigos publicados entre janeiro de 2010 a março de 2016; (f) artigos com um ou mais descritores solicitados; e (g) acesso ao texto completo.
$\mathrm{Na}$ terceira fase, os artigos que atenderam aos critérios de elegibilidade foram pré-selecionados, com base no título e no resumo. Foi verificada a avaliação dos títulos, em que foram incluídos artigos que contivessem, pelo menos, um dos descritores relacionados à temática. Foram considerados os artigos cujos resumos apresentassem informações sobre o desempenho em funções executivas e/ou atenção em crianças e/ou adolescentes com TDAH que estavam ou não usando o metilfenidato e excluídos os que apontassem para o uso do metilfenidato em outra (s) patologia (s) que não fosse o TDAH ou estudos que abordassem o metilfenidato em crianças com TDAH sem relacioná-lo às funções executivas e/ou à atenção. As informações encontradas foram tabuladas por ano de publicação, título, autores, método de pesquisa, objetivos, tipo de amostra (idade, sexo), tamanho da amostra, instrumentos utilizados para analisar as funções executivas, metodologia utilizada para definir a presença do TDAH e principais resultados.

Na última fase, os artigos selecionados depois das análises iniciais foram analisados de forma crítica, para atender ao objetivo proposto e responder à questão geradora.

\section{RESULTADOS E DISCUSSÃO}

Por atenderem ao critério inicial de busca pelos descritores, foram selecionados 71 artigos, dos quais 13 permaneceram no estudo depois da primeira fase de análise, e os demais $(n=58)$ foram excluídos por não atenderem aos critérios estabelecidos pelos seguintes motivos: cinco por serem pesquisas duplicadas (o mesmo artigo surgiu em uma ou mais bases de dados); quatro foram desconsiderados por serem artigos de meta-análise ou revisão sistemática; 11 por serem pesquisas feitas com adultos; quatro, com animais; quatro, por abordarem outros grupos clínicos; e 30 por não contemplarem a temática pesquisada para atingir os objetivos propostos (como mostra a Figura 1). Em seguida, foram excluídos sete artigos, porque envolviam outras variáveis $(n=2)$, amostras de adultos $(n=1)$ e amostras de crianças com outros transtornos $(n=4)$. Por fim, foram selecionados seis artigos que atenderam aos critérios de inclusão estabelecidos, como mostra a Figura 1.

A tabulação realizada para análise dos artigos contém critérios como: autores e ano de publicação, amostra, instrumentos utilizados para atingir o objetivo, objetivo do estudo, e principais resultados que foram encontrados em cada estudo, como mostra a Tabela 1.

Os resultados foram apresentados e discutidos em quatro partes: (a) caracterização da amostra (idade, sexo, tamanho amostral, tipologia do TDAH); (b) procedimentos (uso ou não de grupo-controle, local de seleção da amostra); (c) tipo de instrumentos utilizados para a análise das funções executivas e diagnóstico do TDAH; (d) relação entre o metilfenidato e o desempenho em funções executivas e atenção.

\section{Caracterização e Análise das Amostras dos Estudos Selecionados}

Nas pesquisas analisadas, foram utilizadas amostras de crianças e adolescentes alocados na faixa etária que variou de seis a 16 anos. A maior das amostras apresentou 142 sujeitos, e a menor, com 26 , de ambos os gêneros, exceto a pesquisa de Pasini et al. (2013), que incluiu apenas o sexo masculino e trouxe esse fato como uma limitação do estudo, mas pontuou que, mesmo assim, ele foi importante por ser o primeiro estudo em que foram avaliados os efeitos neuropsicológicos do metilfenidato (MPH) administrado cronicamente em sujeitos com TDAH depois de oito semanas de privação do medicamento.

É válido ressaltar a importância da inclusão de ambos os sexos na pesquisa para evitar a restrição da generalização dos resultados a uma parcela da população, deixando de lado as diferenças de sexo que podem estar presentes na patologia do TDAH, pois, como é sabido, o transtorno acomete mais a meninos do que a meninas, em uma proporção de $2: 1$, e os meninos apresentam comportamentos mais hiperativos, impulsivos e sintomas visualmente notáveis, enquanto as meninas, mais comportamentos de desatenção, o que leva a um maior encaminhamento clínico de garotos (Nardi et al., 2015; Whitbourne \& Halgin, 2015). 
Tabela 1

Características e Principais Resultados dos Artigos Incluidos e que Estavam Indexados em Periódicos com Fator de Impacto que Variou entre 0,425 e 4,450

\begin{tabular}{|c|c|c|c|c|}
\hline Referência & Amostra & Instrumentos & Objetivo & Principais Resultados \\
\hline $\begin{array}{l}\text { Blum, Jawad, } \\
\text { Clarke, e Power, } \\
2011\end{array}$ & $\begin{array}{l}30 \text { pacientes } \\
6-12 \text { anos de } \\
\text { idade }\end{array}$ & $\begin{array}{l}\text { GDS-CPT; TEA-Ch; } \\
\text { DigitSpan; } \\
\text { Finger Windows } \\
\text { Subtest }\end{array}$ & $\begin{array}{l}\text { Investigar o efeito do OROS- } \\
\text { metilfenidato nos componentes da } \\
\text { atenção e funções executivas em } \\
\text { crianças com TDAH }\end{array}$ & $\begin{array}{l}\text { O OROS-metilfenidato, na dose ótima, } \\
\text { melhora a inibição da resposta e tem } \\
\text { efeitos variáveis em domínios de } \\
\text { atenção e funcionamento executivo. } \\
\text { Não houve melhora nas tarefas que } \\
\text { avaliam atenção seletiva e controlada } \\
\text { ou nas duas tarefas que avaliam a } \\
\text { memória de trabalho. }\end{array}$ \\
\hline $\begin{array}{l}\text { Yildiz, Sismanlar, } \\
\text { Memik, Karakaya, } \\
\text { e Agaoglu, } 2011\end{array}$ & $\begin{array}{l}30 \text { pacientes } \\
8-14 \text { anos de } \\
\text { idade }\end{array}$ & $\begin{array}{l}\text { K-SADS, } \\
\text { CGI-S, } \\
\text { CGI-I, } \\
\text { T-DSM-IV-S, } \\
\text { WCST, } \\
\text { Stroop Test, } \\
\text { VMS }\end{array}$ & $\begin{array}{l}\text { Comparar a segurança, eficácia, } \\
\text { tolerabilidade e os efeitos da } \\
\text { atomoxetina (ATX) e OROS- } \\
\text { metilfenidato nas funções } \\
\text { executivas em crianças e } \\
\text { adolescentes com TDAH }\end{array}$ & $\begin{array}{l}\text { O tratamento com ATX foi efetivo } \\
\text { e seguro em uma dose de } 1.2 \mathrm{mg} / \\
\text { dia e, juntamente com OROS-MPH, } \\
\text { produziu significantes melhoras na } \\
\text { gravidade dos sintomas e em FE's, mas } \\
\text { o OROS-MPH forneceu uma melhora } \\
\text { mais acentuada nos sintomas e nas } \\
\text { habilidades de FE's. }\end{array}$ \\
\hline $\begin{array}{l}\text { Yang, Cao, Shuai, } \\
\text { Li, Chan, e Wang, } \\
2012\end{array}$ & $\begin{array}{c}\text { GE: } 142 \text { pacientes } \\
\text { GC: } 46 \text { pacientes } \\
7-14 \text { anos de } \\
\text { idade }\end{array}$ & $\begin{array}{c}\text { ARS, } \\
\text { CGI-S, } \\
\text { Stroop Test, RCFT, } \\
\text { Digit Span, } \\
\text { ToL, } \\
\text { Trail-Making Test, } \\
\text { Verbal Fluency Test }\end{array}$ & $\begin{array}{l}\text { Comparar os efeitos do OROS- } \\
\text { MPH e ATX na melhora das } \\
\text { funções executivas de crianças e } \\
\text { adolescentes com TDAH por um } \\
\text { processo aleatório controlado }\end{array}$ & $\begin{array}{l}\text { Os escores dos testes foram melhores } \\
\text { para o grupo OROS-MPH, mas não } \\
\text { estatisticamente significante. No geral, } \\
\text { ambos os medicamentos melhoraram } \\
\text { as FE's em crianças e adolescentes } \\
\text { com TDAH, embora o OROS-MPH } \\
\text { seja mais efetivo. }\end{array}$ \\
\hline $\begin{array}{l}\text { Huang, Wang, e } \\
\text { Chen, } 2012\end{array}$ & $\begin{array}{l}103 \text { pacientes } \\
6-16 \text { anos de } \\
\text { idade }\end{array}$ & $\begin{array}{l}\text { K-SADS-E; } \\
\text { (TOVA)- Visual }\end{array}$ & $\begin{array}{l}\text { Determinar se há melhora nas } \\
\text { funções neurocognitivas de } \\
\text { pacientes com TDAH durante } \\
12 \text { meses de tratamento com } \\
\text { MPH no estado livre de outros } \\
\text { medicamentos }\end{array}$ & $\begin{array}{l}\text { O MPH melhorou os índices de erros } \\
\text { de comissão e sensibilidade da resposta } \\
\text { avaliados pelo TOVA, mas não houve } \\
\text { melhora nos outros índices deste teste. } \\
\text { Quanto ao desempenho em atenção } \\
\text { sustentada e resposta de velocidade, o } \\
\text { MPH não foi eficaz para melhorá-lo. }\end{array}$ \\
\hline $\begin{array}{l}\text { Yilmaz, Gikcen, } \\
\text { Fettahoglu, e } \\
\text { Ozatalay, } 2013\end{array}$ & $\begin{array}{l}\text { GE: } 30 \text { pacientes } \\
\text { GC: } 30 \text { pacientes } \\
7-12 \text { anos de } \\
\text { idade }\end{array}$ & $\begin{array}{l}\text { K-SADS-PL } \\
\text { WCST } \\
\text { Stroop Test }\end{array}$ & $\begin{array}{l}\text { Avaliar os efeitos do metilfenidato } \\
\text { (MPH) nas FE's em crianças com } \\
\text { subtipos combinados de TDAH }\end{array}$ & $\begin{array}{l}\text { Tratamento com MPH em crianças } \\
\text { com TDAH-C afetou positivamente } \\
\text { sua performance no WCST e Teste } \\
\text { de Stroop, significando mudanças } \\
\text { positivas na perseveração e efeito de } \\
\text { interferência e nomeação de cores. } \\
\text { Mudanças positivas similares foram } \\
\text { observadas em crianças saudáveis } \\
\text { indicando influência do efeito da } \\
\text { prática e processos de aprendizagem. }\end{array}$ \\
\hline $\begin{array}{l}\text { Pasini, Sinibaldi, } \\
\text { Paloscia, Douzgou, } \\
\text { Pitzianti, Romeo, } \\
\text { Curatolo, e Pizzuti, } \\
2013\end{array}$ & $\begin{array}{l}108 \text { pacientes } \\
7-15 \text { anos de } \\
\text { idade }\end{array}$ & $\begin{array}{l}\text { K-SADS-PL, ARS, } \\
\text { CPRS-R:S, } \\
\text { C-GAS } \\
\text { ToL, } \\
\text { CPT II, } \\
\text { N-Back working } \\
\text { memory test }\end{array}$ & $\begin{array}{l}\text { Avaliar o efeito do MPH em } \\
\text { funções neurocognitivas em } \\
\text { particular como: memória de } \\
\text { trabalho, inibição e planejamento }\end{array}$ & $\begin{array}{l}\text { O MPH produziu melhora } \\
\text { nas respostas de inibição e } \\
\text { memória de trabalho em crianças } \\
\text { independentemente do genótipo. }\end{array}$ \\
\hline
\end{tabular}

Nota . GC = grupo controle; GE = grupo experimental; Gordon Diagnostic System-Continuous Performance Test (GDS-CPT); Test ofEverydayAttention for Children(TEA-Ch); Inventário Infantil para Transtornos Afetivos e Esquizofrenia (K-SADS) nas suas versões "epidemiológica" (K-SADS-E) e versão do "presente e duração de vida" (K-SADS-PL); Escala de Impressão Clínica Global (CGI) para "severidade" (CGI-S) e para "aperfeiçoamento" (CGI-I); Turgay Baseada no DSM-IV-Escala de Triagem e Avaliação de Transtornos do Comportamento de Crianças e Adolescentes (T-DSM-IV-S); Teste Wisconsin de Classificação de Cartas (WCST); Teste de Stroop; Palmo de Memória Visual (VMS); Escala de Classificação do TDAH-IV (ARS); Teste Rey de Figura Complexa (RCFT); DigitSpan; Finger Windows Subtest;Trail-Making Test; Teste de Fluência Verbal (VFT); Teste de Variáveis da Atenção (TOVA)-Visual; Conners' Parent Escala de Avaliação Revisada, versão curta (CPRS-R:S), Escala de Avaliação Global das Crianças (C-GAS), Torre de Londres (ToL); Teste de Desempenho Completo (CPT II), Teste N-Back de Memória de Trabalho (N-Back workingmemorytest). 


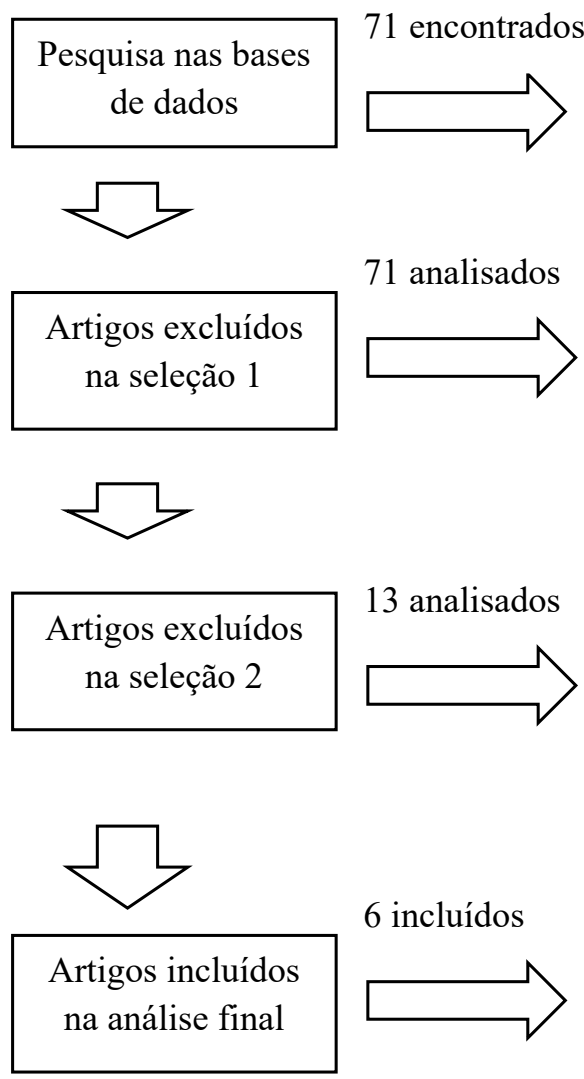

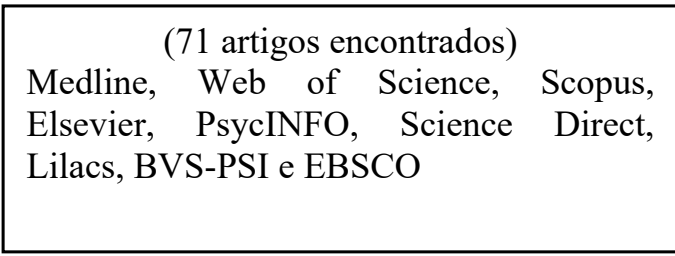

(58 artigos excluídos, 13 incluídos)

Pesquisas duplicados: 5

Artigos de revisão ou meta-análise: 4

Não contemplam o tema: 30

Pesquisas com adultos ou animais: 15

Outros grupos clínicos: 4

(7 artigos excluídos, 6 incluídos)

Estudos envolvendo outras variáveis: 2

Amostras de adultos: 1

Amostras de crianças com outros

transtornos: 4

(6 artigos para a revisão sistemática)

Todos atenderam aos critérios de inclusão estabelecidos em cada uma das etapas.

Figura 1. Fluxograma com Estratégias de Busca para Inclusão dos Artigos da Revisão Sistemática

\section{Análise dos Procedimentos Utilizados nos Estudos Selecionados}

Apenas dois dos artigos analisados (Yang et al., 2012; Yilmaz et al., 2013) dividiram as amostras em grupo-controle e grupo experimental. Os outros não incluíram um grupocontrole de crianças sem o transtorno, o que permitiria que se comparasse o desempenho entre os sujeitos com TDAH com os sujeitos saudáveis nas tarefas avaliadas. Essa foi uma limitação exposta por Huang, Wang, \& Chen (2012) e Yildiz et al., (2011). Porém os outros dois estudos (Blum et al., 2011; Pasini et al., 2013) não citaram o fato como limitação.

É importante que o estudo tenha um grupo-controle para que seja possível analisar se os bons resultados provenientes do uso do medicamento se devem à própria medicação ou à maturação natural dos indivíduos e se há relação do resultado com o efeito de aprendizagem quando o instrumento é reaplicado.

Os estudos basearam-se na classificação estabelecida pelo DSM, que divide o TDAH em três subtipos (combinado, desatento ou hiperativo/impulsivo), em se tratando do DSMIV. Quanto ao DSM-5, o termo subtipo foi alterado para apresentação atual do transtorno, visto que não há evidências de estabilidade no desenvolvimento do sujeito que apoiem o termo "subtipos".

Três dos estudos analisados (Blum et al., 2011; Pasini et al., 2013; Yilmaz et al., 2013) só incluíram crianças com TDAH tipo combinado. Os autores Huang et al. (2012) e Yang et al. (2011) incluíram no estudo crianças e adolescentes dos três tipos de TDAH, e Yildiz et al. (2011), dos tipos combinado e desatento. Quanto a isso, ainda que as medicações sejam igualmente eficazes nas diferentes apresentações do transtorno, os sujeitos com TDAH apresentação desatenta, comparados com os que têm TDAH apresentação combinada, às vezes precisam de menores doses do MPH (Stein et al., 2003). Convém ressaltar a importância de se considerarem as três apresentações do TDAH devido à diferença que existe no quadro de sintomas entre os três grupos. Entre os três tipos de transtorno, os sujeitos com TDAH combinado são os mais comprometidos social e individualmente (Nardi et al. 2015; Stein et al., 2003).

Os sujeitos que compuseram as amostras dos estudos foram recrutados de ambulatórios e hospitais psiquiátricos infantis, o que, de certa forma, denota que previamente receberam o diagnóstico do TDAH. Porém, apenas um estudo (Huang et al., 2012) claramente mencionou que o diagnóstico foi realizado por dois psiquiatras infantis. 


\section{Análise dos Instrumentos Utilizados para Diagnóstico do TDAH}

De acordo com Bolfer (2014) e Graeff e Vaz (2008), um diagnóstico clínico do TDAH deve ser feito por vários profissionais e levando em consideração a história do sujeito e seu comportamento em mais de um contexto. No entanto, para complementar e confirmar o diagnóstico, todos os seis estudos utilizaram escalas e/ou entrevistas semiestruturadas com pais e/ou professores. O Inventário Infantil para Transtornos Afetivos e Esquizofrenia (K-SADS) foi o mais utilizado. Ele é administrado para pais e crianças por um/a psiquiatra infantil treinado/a e é uma entrevista diagnóstica semiestruturada desenvolvida para avaliar a psicopatologia passada e a atual de crianças e adolescentes, de acordo com os critérios do DSM-III e do DSM-IV. Isso possibilita que as pesquisas analisadas excluam comorbidades e façam um levantamento do histórico atual e anterior dos participantes (Huang et al., 2012; Pasini et al., 2013; Yildiz et al., 2011; Yilmaz et al., 2013).

Outros instrumentos utilizados para complementar o diagnóstico foram a escala de impressão clínica global, para analisar a severidade dos sintomas (CGI-S) ou a melhoria ligada ao tratamento (CGI-I); a escala de triagem e avaliação dos transtornos do comportamento em crianças e adolescentes, baseada no DSM-IV (T-DSM-IV-S); a escala de classificação - IV do TDAH (ARS); a escala conners de avaliação revisada para pais, versão curta (CPRS-R:S), e a escala de avaliação global das crianças (C-GAS). Todas essas escalas foram utilizadas adequadamente nesses estudos, como ferramenta auxiliar na avaliação clínica do paciente, o que aumentou a confiabilidade do diagnóstico, visto que o diagnóstico do TDAH é complexo e não deve se basear apenas na história clínica do sujeito (Bolfer, 2014).

\section{Análise dos Instrumentos Utilizados para Analisar as Funções Executivas (FE's)}

Quanto aos instrumentos para avaliar as funções executivas e a atenção avaliadas em cada estudo, foram utilizados o teste Wisconsin de classificação de cartas (WCST); o alcance de memória visual (VMS); o teste de Stroop, o teste de figuras complexas de Rey (RCFT); o digitspan; o teste de trilhas; o teste de fluência verbal (VFT); o teste de variáveis da atenção (TOVA); o teste de torre de Hanoi (ToH); o teste de torre de Londres (ToL); o teste de desempenho contínuo II (CPT II), o teste de memória de trabalho N-Back (N-Back), o teste de desempenho contínuo sistema diagnóstico de Gordon (GDS-CPT), o teste de atenção diária para crianças (TEA-Ch), o subteste finger windows e o teste de codificação.

Dentre os instrumentos supracitados, o mais utilizado foi o teste cor-palavra de Stroop $(n=3)$, que avaliou sensibilidade à interferência, atenção seletiva e controle inibitório (Yang et al., 2012; Yildiz et al., 2011; Yilmaz et al., 2013). Nesse teste, o sujeito responde a elementos específicos de um estímulo, inibe processos atencionais mais automáticos e avalia a supressão do estímulo que distrai a atenção, o que é chamado de efeito de interferência (Oliveira et al., 2014; Yildiz et al., 2011).

Em relação ao teste cor-palavra de Stroop, é importante pontuar algumas de suas limitações, visto que é um teste que depende de leituras mais automatizadas dos examinandos. Assim, a capacidade de leitura e de escolarização formal influencia a validade do teste, que não pode ser aplicado em analfabetos ou disléxicos. Outro fator que interfere no desempenho do teste é a capacidade de perceber e discriminar cores. Por essas razões é que se deve evitar aplicar em crianças que ainda não tenham a leitura automatizada ou um bom conhecimento de cores. Também é preciso saber bem quais os sujeitos que participarão da pesquisa para avaliar o uso ou não do Stroop (cor/palavra) e evitar que a validade do teste seja diminuída. Uma variação do paradigma de Stroop é o teste de cinco dígitos proposto em 2004 por Sedó (Oliveira et al., 2014; Sedó et al., 2015).

Nos estudos de Yildiz et al. (2011) e Yilmaz et al. (2013), foi utilizada a versão turca do teste de Stroop, que conduziu o estudo de normatização do formulário do Teste de Stroop para crianças saudáveis turcas com idades entre seis e 11 anos. Como proposto no estudo de normatização na Turquia, foi usado o tempo para completar o quinto cartão como escore de interferência, e, se o tempo fosse reduzido para essa tarefa, isso seria interpretado como redução no efeito de interferência.

Os outros testes mais utilizados pelos estudos avaliados foram o WCST e o digitspan ordem direta e inversa, para avaliar, principalmente, a flexibilidade cognitiva (FC) e a memória de trabalho (MT) verbal/visual, respectivamente. Os artigos de Yildiz et al. (2011) e Yilmaz et al. (2013) utilizaram a versão turca do teste WCST, e os escores envolveram dois fatores: perseveração e raciocínio.

Constata-se que nem todos os instrumentos utilizados nas pesquisas eram validados, padronizados e/ou normatizados para a população estudada. Por essa razão, é importante usar testes com essas características, devido à maior fidedignidade e possibilidade de generalizar os resultados encontrados. Os testes padronizados dos estudos foram o WCST, o VMS, o digitspan, o de fluência verbal, o N-Back memória de trabalho, o GDS-CPT e o finger windows. Além disso, o mercado nacional e o internacional não dispõem de muitos instrumentos padronizados ou normatizados para a população infantil, o que justifica essa lacuna encontrada nos estudos. Então, são necessárias pesquisas psicométricas.

Em relação às habilidades avaliadas pelos instrumentos em cada estudo, dos seis analisados, apenas um (Blum et al., 2011) avaliou a atenção e as funções executivas (FE's), e das FE's, os autores só avaliaram a MT verbal e a visuoespacial. Quanto aos outros estudos, avaliaram apenas a atenção (Huanget et al., 2012) ou uma ou algumas funções 
executivas (Pasini et al., 2013; Yang et al., 2012; Yildiz et al., 2011; Yilmaz et al., 2013).

Nos sujeitos com TDAH, é importante que sejam avaliados tanto a atenção, em particular e em seus vários componentes, quanto as funções executivas, pois esse é um transtorno caracterizado por alterações no córtex pré-frontal e, consequentemente, déficits no funcionamento executivo e nas funções associadas a essa região do cérebro, além de ser caracterizado pela tríade sintomatológica da desatenção, impulsividade e hiperatividade. A atenção é uma função alterada, e, principalmente, no TDAH há apresentação desatenta e combinada (Fuentes et al., 2014; Menezes et al., 2012).

As FE's constituem um conjunto integrado de habilidades, cuja função é de coordenar, planejar e direcionar o comportamento relativo a metas específicas (Diamond, 2013). Assim, essas funções não são uma unidade única, mas um constructo multidimensional composto de um conjunto de habilidades (p. ex.: planejamento, memória de trabalho, flexibilidade cognitiva, controle inibitório, fluência) que exercem o papel de comando (Dias, 2009; Miyake et al., 2000).

Ressalte-se que, para que seja feita uma avaliação completa e mais robusta das FE's, é preciso que seja considerada a atuação das várias habilidades ou componentes. Sabe-se que essas funções estão interligadas, ou seja, que um bom controle inibitório depende de uma boa memória de trabalho, e a flexibilidade cognitiva depende dos dois componentes anteriores, pois, para que um problema seja interpretado sob uma nova perspectiva, a visão anterior deve ser inibida para que a nova abordagem ao problema possa ser ativada na memória de trabalho. Os diferentes tipos de atenção também estão interligados às funções executivas (Bolfer, 2014; Diaset et al., 2010).

\section{Relação entre o Metilfenidato e o Desempenho em Funções Executivas e Atenção}

Para analisar os resultados sobre a relação entre o metilfenidato (MPH) e o desempenho em funções executivas (FE's) e atenção, os estudos foram divididos em dois grupos, de acordo com a semelhança entre os objetivos que visaram atingir. Os artigos de Yildiz et al. (2011) e Yang et al. (2012) compuseram o primeiro grupo (grupo 1), porquanto ambos compararam os efeitos da atomoxetina (ATX) outro medicamento utilizado principalmente na Europa e na América do Norte para tratar o TDAH - quanto do OROS-MPH - metilfenidato de liberação prolongada - nas funções executivas de crianças e adolescentes com TDAH. O segundo grupo (grupo 2) foi composto pelos estudos de Blum et al. (2011), Huanget et al. (2012), Pasini et al. (2013) e Yilmaz et al. (2013), que objetivaram comparar somente os efeitos do MPH nas funções executivas.

O grupo 1 objetivou comparar os efeitos da ATX e do OROS-MPH nas FE's e na atenção em crianças com TDAH.
Para atingir os objetivos propostos, o primeiro estudo do grupo 1 (Yildiz et al., 2011) selecionou 30 sujeitos com idades entre oito e 14 anos, sendo que 26 completaram a pesquisa; e o segundo estudo (Yang et al., 2012) selecionou 262 crianças com TDAH, com idades entre sete e 14 anos, mas continuaram a pesquisa 142 .

O estudo de Yildiz et al. (2011) não incluiu o grupocontrole, e os sujeitos com TDAH receberam 12 semanas de tratamento com ATX ou OROS-MPH e foram inseridos no grupo de um ou de outro medicamento de forma aleatória. A dose inicial foi de $0,5 \mathrm{mg} / \mathrm{kg} / \mathrm{dia}$ de ATX e $18 \mathrm{mg} / \mathrm{dia}$ de MPH. Depois, aumentou para $1,2 \mathrm{mg} / \mathrm{kg} / \mathrm{dia}$ de ATX e 36 a $54 \mathrm{mg} /$ dia de OROS-MPH, com base nos relatos dos pais e dos clínicos na quarta e na oitava semanas. O estudo de Yang et al. (2012) contou com um grupo-controle de 46 sujeitos sem TDAH, e as crianças com TDAH receberam as mesmas dosagens do OROS-MPH (18mg/dia, que podia aumentar a cada semana para 36 e $54 \mathrm{mg} / \mathrm{dia}$, a depender da resposta do paciente) e da ATX $(0,5 \mathrm{mg} / \mathrm{kg} / \mathrm{dia}$, que foi aumentado para $0,8 \mathrm{mg} / \mathrm{kg} / \mathrm{dia}$, na segunda semana, e $1,2 \mathrm{mg} / \mathrm{kg} / \mathrm{dia}$, na terceira e na quarta), quando comparado com o estudo de Yildiz et al. (2011), também do grupo 1.

Essas semelhanças nas dosagens facilitaram as comparações dos resultados das duas pesquisas. Foi observado que os dois estudos (Yildiz et al., 2011; Yang et al., 2012) não avaliaram o desempenho em tarefas de atenção. Quanto às habilidades de FE's, o estudo de 2011 não avaliou a memória de trabalho (MT) verbal, e o de 2012, a MT espacial. Os resultados encontrados em Yildiz et al. (2011) sugerem que a ATX foi efetiva na dose de 1,2 mg/ $\mathrm{kg} /$ dia e, juntamente com o MPH, diminuiu os sintomas e melhorou o funcionamento executivo dos pacientes. Apesar de as duas medicações serem eficazes, o estudo pontuou o OROS-MPH como o maior provedor de melhorias nos sintomas.

O estudo de Yang et al. (2012) corroborou o de Yildiz et al. (2011), visto que seus resultados também mostraram que, apesar de a ATX e o MPH melhorarem as funções executivas em crianças e adolescentes com TDAH, os escores de todos os testes utilizados foram melhores para o grupo OROSMPH, incluindo memória de trabalho, inibição, flexibilidade, planejamento e fluência, bem como no estudo anterior, que avaliou as mesmas funções, menos a fluência verbal.

Os instrumentos utilizados nos estudos do grupo 1 foram o K-SADS, o CGI-S, o CGI-I, T- o DSM-IV-S, o ARS, o teste de Stroop, o WCST, o RCFT, o DigitSpan, o ToL, o VMS, o teste de trilhas e o teste de fluência verbal. Desses, a CGI-S e o teste de Stroop foram igualmente utilizados tanto por Yildiz et al. (2011) quanto por Yang et al. (2012). A CGI-S, escala de sete pontos, foi utilizada para avaliar a severidade dos sintomas do TDAH, e o Stroop, para avaliar a inibição.

Em relação ao grupo 2, os estudos (Blum et al., 2011; Huang et al., 2012; Pasini et al., 2013; Yilmaz et al., 2013) objetivaram avaliar somente o efeito do metilfenidato ou OROS-MPH (Blum et al., 2011) na melhoria das funções 
executivas (Yilmaz et al., 2013; Pasini et al., 2013), das FE's e da atenção (Blum et al., 2011) ou somente da atenção (Huang et al., 2012) em crianças e adolescentes com TDAH. Os estudos desse grupo incluíram sujeitos cujas idades variaram entre seis e 16 anos, e cuja quantidade na amostra foi bem variada, com 30 pacientes (Blum et al., 2011; Yilmaz et al., 2013), 103 (Huang et al., 2012) ou 108 (Pasini et al., 2013) com TDAH. Apenas um dos estudos (Yilmaz et al., 2013) incluiu o grupo-controle, com 30 sujeitos.

No estudo de Blum et al. (2011), os sujeitos com TDAH receberam, durante duas a três semanas, tratamento com OROS-MPH para determinar a dose ótima. Depois disso, responderam ao TEA-Ch, ao digitspan e ao subteste finger windows, no final da primeira semana de tratamento com placebo e no final da primeira semana de $\mathrm{MPH}$, que poderia ser de $18 \mathrm{mg}$, na primeira semana, ou $36 \mathrm{mg}$, na segunda.

Em Huang, Wang e Chen (2012), o TOVA-Visual foi aplicado três vezes em cada paciente. Na primeira vez, a criança estava há seis meses sem usar medicamento ou nunca o havia usado; depois, os pacientes tomavam MPH duas a três vezes por dia, em dose de $0,3-1,0 \mathrm{mg} / \mathrm{kg}$, e eram avaliados com o TOVA no sexto e no décimo segundo meses depois do tratamento medicamentoso.

No artigo de Yilmaz et al. (2013), o único do grupo 2 que utilizou grupo-controle de crianças sem transtorno, a maioria das crianças com TDAH foi tratada com MPH de liberação imediata (IR-MPH) com uma dosagem de mais ou menos $0,54 \mathrm{mg} / \mathrm{kg} / \mathrm{dia}$, e somente quatro utilizaram MPH de liberação prolongada (OROS-MPH) com uma dosagem de mais ou menos $0,70 \mathrm{mg} / \mathrm{kg} / \mathrm{dia}$. Essas crianças pararam de usar o medicamento durante, pelo menos, 48 horas antes da primeira administração dos testes WCST e Stroop. Depois dessa primeira sessão de testagem, os dois instrumentos foram avaliados novamente, um mês depois do uso do medicamento, sempre uma hora depois da administração matinal do MPH.

Do estudo de Pasini et al. (2013) só participaram meninos. Todos receberam MPH de curta duração, administrado pela manhã e à tarde, a uma dose de 0,5 $\mathrm{mg} / \mathrm{kg} / \mathrm{dia}$. Também receberam treinamento dos pais e psicoterapia. Os participantes foram divididos em grupos de acordo com os genótipos (conjunto formado pelos genes do indivíduo que não se modificam naturalmente) DAT 3'UTR VNTR e foram comparados com as respostas obtidas pelo tratamento com MPH. Segundo os autores, o gene transportador de dopamina (DAT) desempenha um papel crucial na suscetibilidade ao TDAH, e o número variável de repetições em tandem na região não traduzida da extremidade 3' (3'UTR VNTR) do DAT pode influenciar a variabilidade da resposta terapêutica ao MPH no TDAH. Nesse estudo, foram aplicados os testes ToL, CPT-II e o de memória de trabalho N-Back quatro vezes- uma, antes do tratamento, e as outras, 90 minutos após a administração matinal do MPH na quarta, na oitava e na vigésima quarta semana de tratamento.
Quanto aos resultados do grupo 2, dos três que avaliaram as funções executivas (Blum et al., 2011; Pasini et al., 2013; Yilmaz et al., 2013), o de Blum et al. (2011) concluiu que o OROS-MPH em dose ótima melhorou o desempenho em testes de inibição de resposta. Por outro lado, o MPH não foi eficaz em nenhum dos dois testes de memória de trabalho. O medicamento também não melhorou a atenção sustentada e controlada. Portanto, os resultados dos subtestes foram inconsistentes.

Em seu estudo, Yilmaz et al. (2013) concluíram que o MPH em crianças com TDAH tipo combinado (TDAH-C) melhorou o desempenho em testes de funções executivas (FE's). Na segunda aplicação do WCST, o MPH foi eficaz para melhorar o desempenho em quase todos os escores em comparação com a primeira. No Stroop, também na segunda aplicação, o MPH melhorou a habilidade de nomear cores (stroop 3 e 4) e diminuiu o efeito de interferência (stroop 5). Assim, Yilmaz et al. (2013) pontuaram que as melhorias do uso do MPH em crianças com TDAH-C na perseveração e no efeito de interferência podem ter se dado devido à prática dos processos de aprendizagem, pois mudanças similares ocorreram em crianças saudáveis.

No estudo de Pasini et al. (2013), o MPH melhorou o desempenho da inibição de resposta e memória de trabalho nos pacientes com TDAH, independentemente do genótipo. Em geral, os resultados indicaram que crianças com TDAH de genótipo 9/9 e parcialmente 9/10 dão respostas neurobiológicas diferentes ao tratamento de longo prazo com MPH quando comparado com o genótipo 10/10.

No que diz respeito à memória de trabalho, que foi avaliada pelos estudos de Blum et al. (2011) e Pasini et al. (2013), houve divergência nos resultados, pois, enquanto o primeiro estudo encontrou que o OROS-MPH não é eficaz na melhoria dessa habilidade em crianças com TDAH, Pasini et al. (2013) encontraram o contrário. Sobre o controle inibitório, que foi avaliado por Yilmaz et al. (2013) e Pasini et al. (2013), houve concordância dos resultados, visto que ambos encontraram eficácia do MPH na melhoria do controle inibitório, que foi avaliado ou pelo Stroop ou pelo CPT II, respectivamente. Já a flexibilidade cognitiva foi avaliada apenas por Yilmaz et al. (2013), que também constataram eficácia do medicamento na melhoria dessa habilidade.

Quanto à atenção, foi avaliada em todos os seus três componentes por Blum et al. (2011), através do TEA-Ch, e por Huang et al. (2012) foram avaliadas variáveis da atenção através do TOVA. Esses últimos autores encontraram que o MPH melhorou os índices de erros de comissão (quando as informações são interpretadas de forma errada) e sensibilidade da resposta avaliados pelo TOVA, mas não houve melhora nos outros índices desse teste. No que diz respeito ao desempenho em atenção sustentada e resposta de velocidade, o MPH não foi eficaz. O resultado da atenção sustentada foi convergente com o encontrado por Blum et al. (2011). Quanto aos outros resultados, não foi possível comparar os estudos. 
Assim, alguns resultados corroboram, e outros divergem com os de autores como Bolfer (2014), Ferreira (2006) e Ortega et al. (2010), cujos trabalhos falam sobre a eficácia do MPH para que crianças e adolescentes com MPH tenham um desempenho melhor nas funções executivas e na atenção. Quanto aos artigos do grupo 2, que avaliaram desempenho do MPH nas FE's, comparados com os do grupo 1, também sobre os efeitos do MPH, Yilmaz et al. (2013) e Pasini et al. (2013) concordaram com o grupo 1 quanto à melhora do controle inibitório, da flexibilidade cognitiva, do planejamento e da memória de trabalho.

Em relação à ATX avaliada nos artigos do grupo 1, os resultados corroboraram os estudos de Taracena e Rada (2007), em que eles constataram que seu efeito no funcionamento neuropsicológico é incerto, visto que os estudos encontraram mais eficácia do MPH do que da ATX. No grupo 1, as iguais dosagens de ATX e OROS-MPH tornaram mais válidas as comparações dos dois estudos, e os resultados encontrados foram semelhantes e concordaram em relação à maior eficácia do OROS-MPH do que da ATX. Assim, não houve limitações quanto às comparações dos estudos desse grupo.
Por outro lado, apesar de as doses de administração do MPH terem sido similares nos estudos do grupo 2, houve diferença em relação ao tempo em que as crianças e os adolescentes com TDAH já usavam o medicamento ou ao tempo de uso do medicamento que foi estabelecido para a realização das pesquisas. Isso pode ter influenciado os resultados divergentes. Outra limitação, ao comparar os resultados dos grupos, foi o fato de que, apesar da semelhança entre os objetivos, para atingi-los, os autores não usaram todos os instrumentos para avaliar funções executivas (FE's), ou seja, não avaliaram todas as habilidades de FE's nem a atenção, ou só avaliaram a atenção.

Como já foi exposto, é importante avaliar todas as habilidades de FE's e de atenção visto que o TDAH envolve alterações nas regiões responsáveis pelo bom desempenho dessas habilidades e que as FE's constituem um conjunto integrado de habilidades. Quanto à inclusão de diferentes tipos de TDAH em cada estudo, essa não é vista como uma limitação, já que as medicações são igualmente eficazes em todas as apresentações do transtorno, como relatado por Stein et al. (2003).

\section{CONSIDERAÇÕES FINAIS}

O objetivo desta pesquisa foi o de realizar uma revisão sistemática dos estudos que envolvem a verificação do desempenho das funções executivas e atenção em crianças e adolescentes que estavam ou não usando o metilfenidato, a fim de encontrar evidências sobre se havia relação entre a utilização do medicamento e o desempenho em funções executivas e atenção em crianças com TDAH. Para isso, foram considerados as características metodológicas de cada estudo e os resultados encontrados. Como esperado, existem outras substâncias químicas utilizadas como estratégias de tratamento para o TDAH, porém, o metilfenidato é a primeira escolha entre os profissionais médicos.

Os resultados sobre a relação do uso do metilfenidato e o desempenho em funções executivas e atenção foram controversos. Em um dos estudos, não foi encontrada relação forte, que possibilite a indicação de eficácia entre o uso do metilfenidato e o desempenho em memória de trabalho verbal e visuoespacial nem nas atenções sustentada e controlada. Em outros estudos, foi encontrada relação positiva com o controle inibitório, a flexibilidade cognitiva e a fluência verbal.

Assim, os resultados não foram convincentes para comprovar a relação proposta. Em parte, esses resultados inconclusivos podem ser justificados pelos diferentes métodos utilizados nos estudos, principalmente no que se refere aos instrumentos para analisar o desempenho das funções executivas, porque nem todos eram validados para a população estudada, à escolha de apenas uma função executiva para a análise e ao diagnóstico do TDAH.

É importante ressaltar que, em geral, os estudos selecionaram a amostra clínica em clínicas e hospitais. Entretanto, alguns estudos consideraram apenas uma apresentação do TDAH. Além disso, variáveis como tempo de uso da medicação, dosagem e nível socioeconômico da família não foram consideradas e/ou controladas, e a maioria das amostras dos estudos não era representativa da população, o que pode implicar baixo poder estatístico para se fazer inferências e generalizações.

Os estudos sobre funções executivas estão em fase de crescimento, e muitas informações novas surgem em cada artigo. No entanto, percebe-se algum consenso no que se refere aos instrumentos utilizados em crianças e adolescentes, apesar de o número de eficácia comprovada ainda ser escasso. Isso pode justificar, em parte, o número de pesquisas que só analisaram um tipo de função executiva. Por essa razão, são necessários mais instrumentos padronizados no Brasil que avaliem funções executivas em crianças.

Como já exposto, o uso do metilfenidato apresentou relação com o desempenho de algumas funções executivas e atenção, porém os estudos não são conclusivos. Finalmente, sugere-se que sejam feitos novos estudos que levem em consideração critérios protocolares para selecionar a amostra clínica e controlar variáveis como tempo e dosagem de utilização da medicação. Também é relevante considerar os instrumentos empregados para analisar as funções executivas e o tipo de função que estará sendo investigada.

Estima-se que novas pesquisas sobre a temática possam contribuir para melhorar a qualidade de vida de crianças e adolescentes com TDAH, investigar e esclarecer sobre os efeitos do metilfenidato nas funções executivas e na atenção. Acredita-se, ainda, que investigações longitudinais possam ser realizadas para fortalecer a compreensão sobre a relação ao longo da vida. 


\section{REFERÊNCIAS}

Agência Nacional de Vigilância Sanitária. (2014). Metilfenidato no tratamento de crianças com transtorno de déficit de atenção e hiperatividade. Boletim Brasileiro de Avaliação de Tecnologias em Saúde, 8(23), 1-18. https://docs.bvsalud.org/ biblioref/2021/04/1178586/brats23.pdf

American Psychiatric Association. (2013). Diagnostic and statistical manual of mental disorders (DSM-5) (5a ed.). American Psychiatric Association.

Andrade, E. R., \& Scheuer, C. (2004). Análise da eficácia do metilfenidato usando a versão abreviada do questionário de conners em transtorno de déficit de atenção/hiperatividade. Arquivos de Neuro-Psiquiatria, 62(1), 81-85.10.1590/S0004282X2004000100014

Bezerra, C. S. G. B. (2014). Interferência do cloridrato de metilfenidato no desempenho de escolares com transtorno de déficit de atenção/hiperatividade[Tese de Doutorado, Universidade Federal de Goiás]. Biblioteca Digital de Teses e Dissertações da UFG. https://repositorio.bc.ufg.br/tede/ handle/tede $/ 4017$

Blum, N. J., Jawad, A. F., Clarke, A. T., \& Power, T. J. (2011). Effect of osmotic-release oral system methylphenidate on different domains of attention and executive functioning in children with attention-deficit-hyperactivity disorder. Developmental Medicine \& Child Neurology, 53, 843-849. 10.1111/j.14698749.2011.03944.x.

Bolfer, C. P. M. (2009). Avaliação neuropsicológica das funções executivas e da atenção em crianças com transtorno do déficit de atenção/hiperatividade (TDAH)[Dissertação de Mestrado, Faculdade de Medicina de São Paulo]. Biblioteca Digital da USP.10.11606/D.5.2009.tde-09022010-171726

Bolfer, C. P. M. (2014). Avaliação neuropsicológica das funções executivas e da atenção antes e depois do uso do metilfenidato em crianças com transtorno do déficit de atenção/ hiperatividade[Tese de Doutorado, Faculdade de Medicina de São Paulo]. Biblioteca Digital da USP. 10.11606/T.5.2014. tde-24022015-115036

Brant, L. C., \& Carvalho, T. R. F. (2012). Metilfenidato: Medicamento gadget da contemporaneidade. Interface Comunicação, Saúde, Educação, 16(42), 623-636.10.1590/ S1414-32832012000300004

Capovilla, A. G. S., \& Dias, N. M. (2008). Desenvolvimento de habilidades atencionais em estudantes da $1^{\mathrm{a}}$ à $4^{\mathrm{a}}$ série do ensino fundamental e relação com rendimento escolar. Revista de Psicopedagogia, 25(78), 198-211. http://pepsic.bvsalud.org/ $\mathrm{pdf} / \mathrm{psicoped} / \mathrm{v} 25 \mathrm{n} 78 / \mathrm{v} 25 \mathrm{n} 78 \mathrm{a} 03 . \mathrm{pdf}$

Diamond,A.(2013). Executive functions. Annual Review of Psychology, 64, 135-168.10.1146/annurev-psych-113011-143750

Dias, N. M. (2009). Avaliação neuropsicológica das funções executivas: Tendências desenvolvimentais e evidências de validade de instrumentos [Dissertação de Mestrado, Universidade Presbiteriana Mackenzie].Biblioteca Digital Mackenzie. http://tede.mackenzie.br/jspui/handle/tede/1746

Dias, N. M., Menezes, A., \& Seabra, A. G. (2010). Alterações das funções executivas em crianças e adolescentes. Estudos Interdisciplinares em Psicologia, 1(1), 80-95.10.5433/2236-6407.2010v1n1p80

Ferreira, J. T. C. (2006). Transtorno de déficit de atenção e hiperatividade (TDAH): Avaliaçãoneuropsicológica das funções frontais em crianças e adolescentes antes $e$ após tratamento com metilfenidato[Tese de Doutorado, Universidade Federal do Espírito Santo].Biblioteca Digital de Teses e Dissertações da UFES. http://portais4.ufes.br/ posgrad/teses/nometese 383 Tese $\% 20 J a n e \% 20$ Tagarro $\% 20$ Corr\%EAa\%20Ferreira.pdf
Fuentes, D., Malloy-Diniz, L. F., Camargo, C. H. P., \& Consenza, R. M. (Orgs.).(2014). Neuropsicologia: Teoria e prática (2a ed.). Artmed.

Gonçalves, H. A., Mohr, R. M., Moraes, A. L., Siqueira, L. S., Prando, M. L., \& Fonseca, R. P. (2013). Componentes atencionais e de funções executivas em meninos com TDAH: Dados de uma bateria neuropsicológica flexível. Jornal Brasileiro de Psiquiatria, 62(1), 13-21.10.1590/S004720852013000100003

Graeff, R. L., \& Vaz, C. E. (2008). Avaliação e diagnóstico do transtorno de déficit de atenção e hiperatividade (TDAH). Psicologia USP, 19(3), 341-361.10.1590/S010365642008000300005

Huang, Y., Wang, L., \& Chen, C. (2012). Long-term neurocognitive effects of methylphenidate in patients with attention deficit hyperactivity disorder, even at drug-free status. $B M C$ Psychiatry, 12(194), 1-7.10.1186/1471-244X-12-194

Itaborahy, C., \& Ortega F. (2013). O Metilfenidato no Brasil: Uma década de publicações. Ciência \& Saúde Coletiva, 18, 80316.10.1590/S1413-81232013000300026

Maneeton, N., Maneeton, B., Woottiluk, P., Suttajit, S., Likhitsathian, S., Charnsil, C., \& Srisurapanont, M. (2015). Comparative efficacy, acceptability, and tolerability of dexmethylphenidate versus placebo in child and adolescent ADHD: A meta-analysis of randomized controlled trials. Neuropsychiatric Disease and Treatment, 11, 2943-2952. 10.2147/NDT.S91765

Menezes, A., Godoy, S., Teixeira, M. C. T. V., Carreiro, L. R. R., \& Seabra, A. G. (2012). Definições teóricas acerca das funções executivas e da atenção. Em A. G. Seabra, N. M. Dias (Orgs.), Avaliação neuropsicológica cognitiva: Atenção e funções executivas (pp.34-41). Memnon.

Miyake, A., Friedman, N. P., Emerson, M. J., Witzki, A. H., Howerter, A., \& Wagner, T. D. (2000). The unity and diversity of executive functions and their contributions to complex "frontal lobe" tasks: A latent variable analysis. Cognitive Psychology, 4l(1), 49-100. 10.1006/ cogp.1999.0734

Murray, D. W., Childress, A., Giblin, J., Williamson, D., Armstrong, R., \& Starr, H. L. (2011). Effects of OROS methylphenidate on academic, behavioral, and cognitive tasks in children 9 to 12 years of age with attention-deficit/ hyperactivity disorder. Clinical Pediatrics, 50(4), 308320.10.1177/0009922810394832

Nardi, A. E., Quevedo, J., \& Silva, A. G. (2015). Transtorno de déficit de atenção/hiperatividade: Teoria e clínica. Artmed.

Oliveira, T. D., Malloy-Diniz, L. F., Magalhães, S., Costa, D. S., Lacerda, S. R., Querino, E. H. G.,... Paula, J. J. (2014, 16 de Maio - 17 de Maio). Propriedades psicométricas do Teste dos Cinco Dígitos para o contexto brasileiro: Estudo preliminar com a população adulta[Relato de Pesquisa], I Congresso da Sociedade Brasileira de Neuropsicologia Jovem, Belo Horizonte, MG, Brasil. 10.13140/RG.2.1.4737.9043

Ortega, F., Barros, D., Caliman, L., Itaborahy, C., Junqueira, L., \& Ferreira, C. P. (2010). A ritalina no Brasil: Produções, discursos e práticas. Interface - Comunicação, Saúde, Educação, 14(34), 499-510.10.1590/S1414-32832010005000003

Pasini, A., Sinibaldi, L., Paloscia, C., Douzgou, S., Ptzianti, M. B., Romeo, E., Curatolo, P., \& Pizzuti, A. (2013). Neurocognitive effects of methylphenidate on ADHD children with different DAT genotypes: A longitudinal open label trial. European Journal of Paediatric Neurology, 17, 407-414.10.1016/j. ejpn.2013.02.002 
Pires, E. U. (2014). Desenvolvimento de um instrumento computadorizado para avaliar habilidades executivas em crianças: O jogo das cartas mágicas[Tese de Doutorado, Pontifícia Universidade Católica do Rio de Janeiro]. Coleção Digital Maxwell. https://www.maxwell.vrac.puc-rio. br/35687/35687_1.PDF

Posner, M. I. (2012). Imaging attention networks. Neuroimage, 61(2), 450-456. 10.1016/j.neuroimage.2011.12.040

Rohde, L. A., \& Halpern, R. (2004). Transtorno de déficit de atenção/ hiperatividade: Atualização. Jornal de Pediatria, 80(2), 61-70. 10.1590/S0021-75572004000300009

Schoemaker, K., Mulder, H., Dekovic, M., \& Matthys, W. (2013). Executive functions in preschool children with externalizing behavior problems: A meta-analysis. Journal of Abnormal Child Psychology, 41, 457-471.10.1007/s10802-012-9684-x

Sedó, M. A., Paula, J. J., \& Malloy-Diniz, F. (2015). O teste dos cinco dígitos. Hogrefe CETEPP.

Silva, A. C. P., Luzio, C. A., Santos, K. Y. P., Yasui, S., \& Dionísio. G. H. (2012). A explosão do consumo de ritalina. Revista de Psicologia da UNESP, 11(2), 44-57. http://hdl.handle. net/11449/127245

Stein, M. A., Sarampote, C. S., Waldman, I. D., Robb, A. S., Conlon, C., Pearl, P. L., Black, D. O., Seymour, K. E., \& Newcorn, J. H. (2003). A dose-response Study of OROS methylphenidate in children with attention-deficit/hyperactivity disorder. Pediatrics, 112(5), 404-413. 10.1542/peds.112.5.e404

Storebo, O. J., Ramstad, E., Krogh, H. B., Nilausen, T. D., Skoog, M., Holmskov, M.,...Gluud, C. (2015). Methylphenidate for children and adolescents with attention deficit hyperactivity disorder (ADHD). Cochrane Database of Systematic Reviews (Online), 11(CD009885). 10.1002/14651858.CD009885.pub2

Taracena, M. T. L., \& Rada, F. M. (2007). Atomoxetina: Luces y sombras. Psiquiatria Biológica, 14(1), 13-23. https://pesquisa. bvsalud.org/portal/resource/pt/ibc-053001

Toassa, G. (2012). Sociedade tarja preta: Uma crítica à medicalização de crianças e adolescentes. Fractal: Revista de Psicologia, 24(2), 429-434.https://periodicos.uff.br/fractal/article/ view/4905

Whitbourne, S. K., \& Halgin, R. P. (2015). Psicopatologia: Perspectivas clínicas dos transtornos psicológicos ( $7^{\mathbf{a}}$ ed.). AMGH.

Yang, L., Cao, Q., Shuai, L., Chan, R. C. K., \& Wang, Y. (2012). Comparative study of OROS-MPH and atomoxetine on executive function improvement in ADHD: A randomized controlled trial. International Journal of Neuropsychopharmacology, 15, 15-26. $10.1017 / \mathrm{S} 1461145711001490$

Yildiz, O., Sismanlar, S. G., Memik, N. C., Karakaya, I., \& Agaoglu, B. (2011). Atomoxetine and methylphenidate treatment in children with ADHD: The efficacy, tolerability and effects on executive functions. Child Psychiatry \& Human Development, 42, 257-269.10.1007/s10578-010-0212-3

Yilmaz, A., Gokcen, C., Fettahoglu, E. C., \& Ozatalay, E. (2013). The effect of methylphenidate on executive functions in children with attention-deficit hyperactivity disorder. Bulletin ofClinicalPsychopharmacology, 23(2), 162-170. 10.5455/ bcp. 20121130090251 